\title{
Roaming Scenarios Based on SIP
}

\author{
Andrea Floris ${ }^{1}$ and Luca Veltri ${ }^{1}$ \\ ${ }^{1}$ CoRiTeL ñ Research Consortium on Telecommunication, \\ Via Anagnina 203, 00040 Roma, Italy \\ \{floris, veltri\}@coritel.it
}

\begin{abstract}
This work deals with the problem of IP mobility amongst different access domains. In particular the problem of the integration of the SIP (Session Initiation Protocol) signaling within a roaming environment is considered. Although SIP natively supports only user mobility, it can be also used to manage full terminal mobility. In this work different SIP registration scenarios for the so-called Pre-call terminal mobility are identified and described in detail. The general aim is allowing mobile users to remain reachable under the same application-layer identifier as terminals change IP address and thus while roaming. In the proposed scenarios the problem of service control from both home and foreign domains has been also considered.
\end{abstract}

\section{Introduction}

The rapid growth of the Internet and the increasing demand for ubiquitous mobile wireless services are the driving forces behind intense activities towards the design of all IP wireless networks in the main international standardization groups. The aim is the definition of an all IP wireless environment that allows roaming users to access integrated data, voice and multimedia services of the Internet via their wireless IP terminals or appliances.

The Session Initiation Protocol (SIP) [1] has been selected, within the IETF, as signalling protocol to set-up, maintain and teardown user calls, to perform complete session management, and to provide user and/or service portability/mobility.

As far as terminal mobility support is concerned, different protocols/mechanisms can be used in combination with specific access network procedures. For instance, a possible solution could be to implement mobility support at network layer, by using the Mobile IP architecture/protocol [2][3]. The primary advantage of using a network layer protocol for terminal mobility is that it can support efficiently applications that are not ì mobility awareî (e.g. normal TCP-based applications). However, some disadvantages of this approach are:

- increased terminal complexity because of the use of multiple protocols for terminal, service and personal mobility;

- use of different protocols and network entities to perform similar functions; for instance, in case of a Mobile IP and SIP combination [4], wireless users use Mobile IP registration and Home Agent or Foreign Agent [2], while wireline users

The original version of this chapter was revised: The copyright line was incorrect. This has been corrected. The Erratum to this chapter is available at DOI: 10.1007/978-3-540-45812-8_28 
utilize SIP REGISTER messages and SIP Registrar servers [1] for similar functions;

- Mobile IP suffers of the well-known problem of triangular routing, i.e. a packet to a mobile host always passes via the home agent, before reaching the terminal [2]; in general, no direct route is used between the mobile node and the corresponding node.

An application layer mobility support may overcome some of the Mobile IP disadvantages. In particular SIP could be used to support terminal, service as well as personal mobility [5][4|[6]. In this way, in addition to the overcoming of the triangular routing and IP encapsulation associated with Mobile IP, some other important advantages arise. First, the minor complexity of terminals, which could use only one protocol for all kinds of mobility; second, SIP mobility easily interacts with preexisting IETF protocols for host configuration, authentication, etc (such as AAA, DHCP or DRCP, SDP, RTP/UDP). Moreover, it might reduce the time required for the registration process.

This work analyses the SIP mobility support for wireless network architectures. We only refer to the so-called ì pre-call mobilityî [7], i.e. the case in which the user moves before starting new calls. Different userís registration cases are here analyzed and illustrated. The proposed SIP-based roaming model is also compliant with the 3GPP signaling architecture [3].

This paper is structured as follows. In section 2 a brief description of the Session Initiation Protocol is given, with particular attention to mobility aspects. In section 3 a general architecture with SIP mobility support is presented. In section 4 several different SIP registration scenarios for a roaming user are proposed and described in detail. Finally some conclusions are given in section 5 .

\section{The Session Initiation Protocol}

In this section we introduce the Session Initiation Protocol. Particular attention is given to functionalities that are concerned with mobility and location update procedures.

\subsection{SIP Overview}

The Session Initiation Protocol (SIP) [1] is an application-layer control protocol that can establish, modify, and terminate multimedia sessions in an IP-based environment. In particular, SIP provides different capabilities such as: i) User location: determination of the end system to be used for communication; ii) User availability: determination of the willingness of the called party to engage in communications; iii) User capabilities: determination of the media and related parameters to be used; iv) Session set-up: "ringing", establishment of session parameters at both called and calling party; v) Session management: including transfer and termination of sessions, modifying session parameters and invoking services. 
Moreover, SIP transparently supports name mapping and redirection services, which allows personal mobility; i.e. users can maintain a single externally visible identifier (SIP URI) and be reachable, regardless of their network location.

\subsection{SIP Registration Mechanism}

SIP is a protocol that offers discovery capability. Each time a SIP user starts a session, SIP discovers the current host(s) on which the called user is reachable. This discovery process is accomplished by the use of SIP proxy, redirect and registrar servers, which are responsible for receiving a request, determining where to send it based on knowledge of the location of the user, and then relay the request. The user location is performed by consulting a location service, which provides address bindings for a particular domain. These address bindings map an incoming SIP URL, in the form of sip:user@domain, to one or more SIP URLs which refer to the current location of the user.

There are many ways by which the content of the location service can be established. SIP also provides its own mechanism that allows a user agent to explicitly create a binding in the location service. This mechanism is known as registration.

The process of registration entails sending a REGISTER message [1] to a special type of SIP server known as a Registrar. The registrar acts as a front end to the location service for a domain, creating, modifying and deleting URLs mappings based on user registration requests.

Generally, a SIP registrar is expected to resolve URLs for its local domain. When a registrar server receives a request for a user registered within its domain, it relays the request to the contact addresses registered for that specified user.

\subsection{Mobility Support with SIP}

The SIP REGISTER request is the core mechanism for supporting personal, service and pre-call mobility [1][5][4][5]. In particular:

i) Personal mobility is the ability for a user to be reachable under the same identifier while using different terminals and independently of its current location, thus while roaming.

ii) Service mobility refers to the ability to obtain the same services regardless of where a user may be roaming. A related aspect of service mobility is the ability to maintain the same set of services when changing providers or proxies.

iii) Pre-call terminal mobility describes the ability of a terminal to dynamically acquire IP addresses, but remain reachable under the same application-layer identifier.

In the following we focus on the Pre-call terminal mobility since it could be the basis for roaming scenarios entirely based on SIP. The registration procedure is still the basic mechanism for supporting such kind of mobility. 


\section{SIP Roaming Model}

In this section a general architecture for mobility support with SIP, for next generation wireless networks, is introduced and described. We will mainly focus on the components involved for supporting the so-called Pre-call terminal mobility.

\subsection{Reference Architecture and Logical Entities}

Let us consider a network architecture composed by several administrative domains, and let us consider the case of a mobile user that moves with his/her terminal between two different domains (Fig. 1).

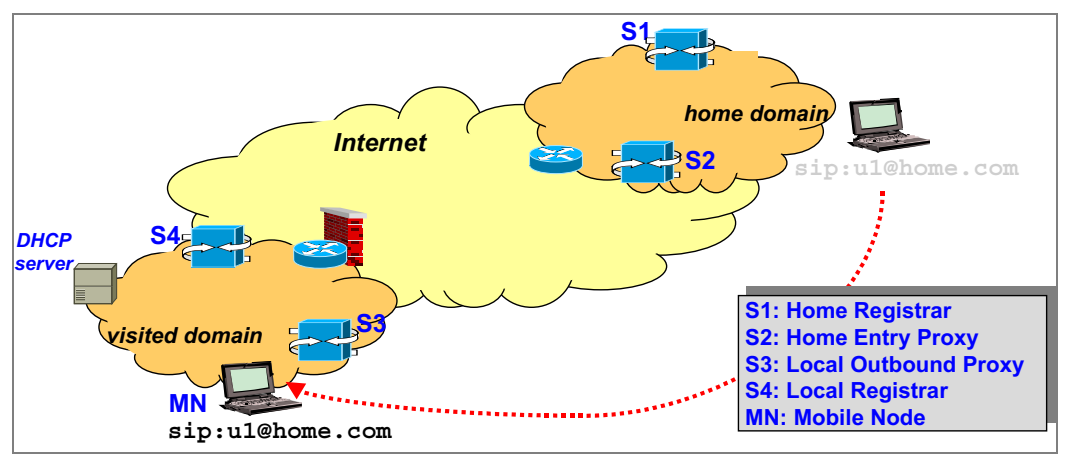

Fig. 1. SIP Roaming: reference architecture

The following entities are considered:

Mobile Node (MN): it is the user mobile terminal that allows users to communicate, and also provides means of interactions and control between users and the network.

The MN updates its IP address as it roams between subnets, either within the same administrative domain or in different domains. As a MN moves (i.e., it changes point of attachment to the network), it needs to acquire a new IP address, possibly a new default gateway/router, subnet mask, other network parameters and information.

All MNs have SIP user agents that provide means of interactions with the SIP servers within the network. We assume that a MN has been permanently assigned a constant identifier by its own domain and is allowed to maintain it regardless of its point of attachment to the network (e.g., while changing its IP address). A user maintains a universal SIP URL anywhere he is roaming. Such identifier may be, for example, embedded by configuration into the communications device (e.g., for an Ethernet phone, personal laptop or workstation) or associated temporarily with a device by some token carried by the user (examples of such tokens include SIMs ñ Subscriber Identifier Module, and smart-cards).

This address implies a home domain and thus at least an Entry Proxy, derived by the DNS SRV lookup of the host address field of the SIP URI. In addition, each device has at least a temporary IP address, which can be used to identify it during a session. 
In some cases, the temporary address might not be directly reachable, for example due to the presence of firewalls and/or network address translators.

Home Registrar: it is a SIP server in the home network that behaves as a registrar for the mobile user: it accepts registration requests and performs request message redirection and/or forwarding. Moreover, it carries out the session control service for the mobile user: it maintains a session state as needed by the network operator for supporting of the user services. In order to carry out its functionalities, the Home Registrar maintains a database with information concerning usersí profiles and details, needed to support mobility management and service control.

Home Entry Proxy: it is the first contact point within the home network for all SIP request messages destined to a subscriber of that domain (operator), or a roaming subscriber currently located within that network operatorís service area. There may be multiple entry proxies within an operatorís network. As far as the SIP registration procedure is concerned, the main functions performed by the Entry Proxy are:

- assigning a SIP registrar to a user performing SIP registration;

- forwarding a SIP Register request, received from another network, towards the selected registrar.

The Entry Proxy is mostly a load-balancing node and can also be used to hide configuration, capacity, and topology of the network from the outside.

Moreover, the Entry Proxy may solve the problem of how a MN discovers its home registrar as it is roaming in a visited network. In this way a user cannot be tied to any particular SIP server.

Local Outbound Proxy: it is the first point of contact (at SIP signalling level) for the mobile user that is roaming within a foreign domain. All SIP messages, sent and received by the roaming user, should cross the proxy. As far as the SIP registration procedure is concerned, the main functions performed by the Outbound Proxy are:

- forwarding the SIP REGISTER request received from the mobile user to the Entry Proxy of the mobile userís home network (determined using the home domain name);

- optionally starting the registration of the mobile user with the local domain (in this case, it has to select the right SIP local registrar).

Local Registrar: it is a SIP server in the local visited domain that can behave as a registrar for the roaming mobile users: it accepts REGISTER request messages. Moreover, it could optionally perform session control service for the mobile user, according to roaming agreements with the home domain. In other words, it may act as a local serving SIP proxy for the roaming users. The registration within the currently visited domain may improve the control of ongoing/incoming sessions.

DHCP Server: itís the entity that provides the means for the terminal configuration. A MN should receive a new IP address and other network information (default router, DNS, outbound proxy, etc.) as soon as it roams into a new network (intra-domain roaming) or a new administrative domain (inter-domain roaming). A DHCP server provides such information [3] [10]. The MN configuration process follows the basic operation of DHCP [3]. There are also some proposals for dynamic host configura- 
tion that are more tied for mobile scenarios than DHCP. A proposal appeared as internet draft is DRCP [11]. DRCP offers functionality to configure a MN on a foreign domain and to provide basic roaming service. DRCP, with respect to DHCP, does not perform address collision resolution process, and it tries to reduce the configuration delay.

\section{Registration Scenarios for Roaming Users}

In this section different SIP registration scenarios are identified and described. Let us consider a mobile user that attaches for the first time to a new visited domain and starts a SIP registration procedure in order to register with his/her Home Registrar updating his/her point of presence (local URL or IP address).

The home network should always maintain the control of end usersí sessions and services regardless of whether the user is at home or in a visited network. In order to support user/service/terminal mobility, mobile users always register with their home networks.

Local registration within the current visited domain can be also performed, in order to deal with some issues as firewall filtering, AAA, etc., and to improve service and networkës performances.

Itís worth to notice that in the considered roaming environment (Fig. 1) where the user moves into a visited network different from its own home domain, AAA (Authentication, Authorization and Accounting) functionalities are expected. The local domain should perform an access control procedure in order to restrict the usage of network resources and services only to authorize users, according to roaming agreements. Thus there is the need to tie the SIP architecture to a general AAA infrastructure and to carry out the SIP registration together with the AAA procedure [12].

However, the SIP-AAA interworking does not change the basic SIP registration signalling, but only introduces some extra steps. Therefore, for simplicity, in the following we will not refer to AAA functionalities.

Now let us consider five roaming scenarios:

\subsection{Basic Home Registration}

In this case (see Fig. 2), the mobile user simply acquires a local IP address in the visited network (for instance from a DHCP server) and sends a SIP REGISTER message towards its home domain, with a SIP Contact header indicating that address. This message has the following main fields:

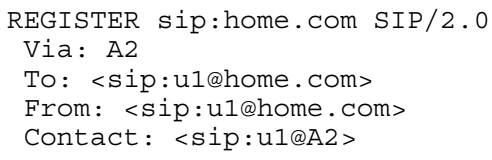

where home.com is mapped to s2.home.com that is the Home Entry Proxy for home.com, and $A 2$ is the local IP address in the foreign domain. 
The request reaches the Home Entry Proxy that dynamically selects the right SIP Registrar for the mobile user and forwards the registration to it. The Entry Proxy does not maintain any state information about the user.

The REGISTER message that arrives to the selected Home Registrar is of the form of:

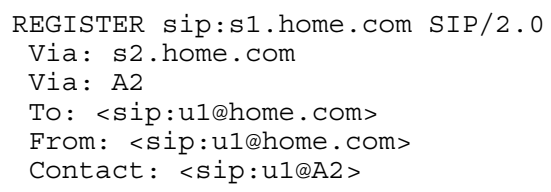

This scenario is very simple and involves only few SIP entities. It follows the basic SIP registration rules [1].

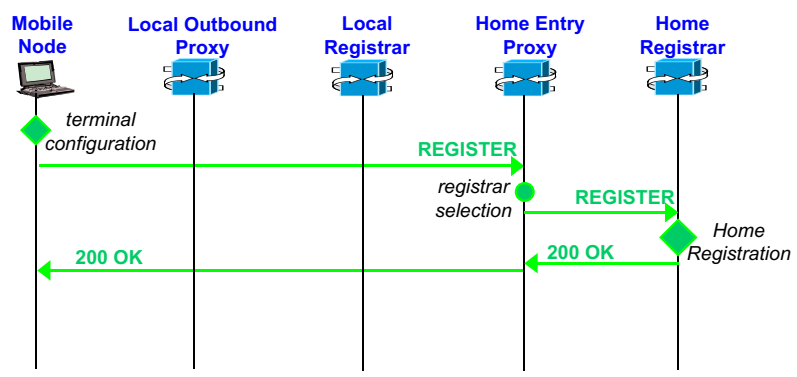

Fig. 2. Basic Home Registration

It makes no difference whether the visited network provides SIP services or not. A Local Outbound Proxy (S3 in Fig. 1) can be used, but it simply forwards the REGISTER request to the home domain.

This approach only works if the visited network does not use any firewall or policy to restrict the access service. Moreover, each movement inside the same visited domain (that implies IP addressís change) requires a new SIP registration message to be sent towards the home domain, for location update at the home registrar. Itís evident that, in order to overcome these drawbacks, a local registration should be expected/required. This approach will be considered in the following scenarios.

\subsection{Third-Party Local Registration}

A third-party registration occurs when the party sending the registration request is not the party that is being registered. In this case, the From header will contain the URL of the party submitting the registration on behalf of the party identified in the To header. In other words, an entity, different from the registering user, acts as User Agent for the latter.

\section{a) Proxy Initiated}

In this case (see Fig. 3) the Local Outbound Proxy intercepts the REGISTER request and any other outgoing SIP messages of the mobile user and changes the address in 
the Contact field into a SIP identifier, assigned to the mobile user within the visited domain.

The Local Proxy first forces the registration of the roaming user with the local domain. For this aim it creates a new SIP REGISTER message and sends it to a local registrar, properly selected (for example s4.visited.com). Moreover, it has to create a new temporary user identifier (for example ì u2@s4.visited.comî) that allows the identification of the user in the local domain. Itís worth to note that the From header of the local REGISTER message contains the URL of the party submitting the registration request on behalf of the party identified in the To header and thus it must contain the Local Outbound Proxyís address (it is a third-party registration).

Itís important that the Local Proxy generates itself the SIP REGISTER message for the registration in the local domain, without any explicit intention from the user, which is therefore completely unaware of the local registration procedure.

The user sends a SIP REGISTER message to its own home domain, as in the Basic Home Registration case.

The Outbound Proxy builds the following SIP message, for local registration:

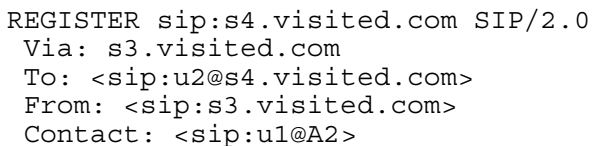

In the simplest case the local identifier for the mobile user can be built as:

u2=u1\#home.com (i.e. u2@s4.visited.com=u1\#home.com@s4.visited.com).

After the reception of the $200 \mathrm{OK}$ for the local registration, the proxy forwards the original SIP REGISTER message towards the home domain. This message has the following main fields:

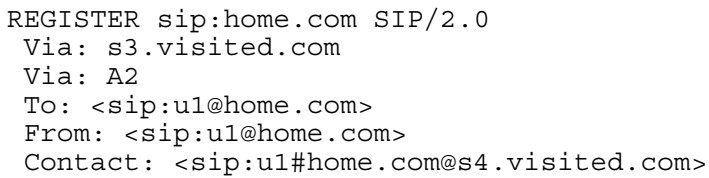

The Contact header has been modified and contains the address of the local registrar (s4.visited.com), according to the local registration.

As in the previous scenario, this message reaches the Home Entry Proxy (s2.home.com) that dynamically selects the right SIP Registrar and forwards the registration to it.

This approach has the advantage that it forces incoming requests to use the local registrar in the foreign domain. Such procedure should be required in network scenarios that implement firewall between access domain and the rest of Internet. In this cases, a local registration can be used to open an entry in the firewall for both the signalling and voice/audio flows. In the considered scenario, all successive movements of the MS within the visited domain requiring new registrations for the mobile user (new IP addresses), are managed locally, without participation of the home domain. In this way there is an improvement of the location-update procedure and better use of network resources; registration with the home domain is only required for expiration prevent or for inter-domain roaming. Besides that, local registration allows the control of multimedia services to be transferred into the current visited domain. 


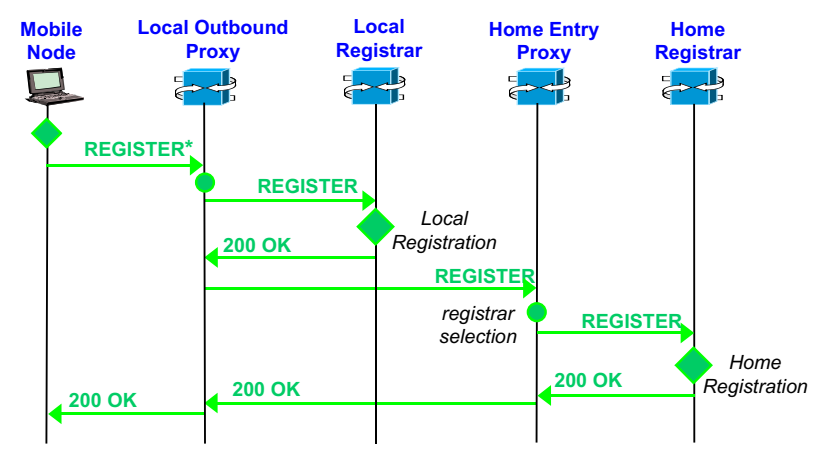

Fig. 3. Proxy-initiated Local Registration

A problem however can arise if some authentication mechanisms are used between the mobile user and its home domain. In fact, in this case, the Contact field can be protected and thus it cannot be modified by the Outbound Proxy, otherwise authentication would be lost. Moreover, if any cryptographic algorithm is used, between the mobile user and its home domain to protect the signalling, the local proxy cannot even read the message.

\section{b) User Initiated}

This scenario is similar to the previous one and the message flow chart is the same (Fig. 3). The only difference is the fact that, this time, the user implicitly asks the Outbound Proxy to execute the local registration for itself.

This implies that the user has to:

- know which new identifier should be used locally (e.g. u2@visited.com);

- know which local registrar he is going to be registered to;

- indicate both these pieces of information in the SIP REGISTER message.

The easiest way to allow the user to get the above information is placing them within ad-hoc DHCP options [10]. During the terminalís configuration procedure, the user, besides the IP address and other local networkís parameters, receives the information for the SIP registration procedure.

The REGISTER message sent by the mobile user is therefore in the form of:

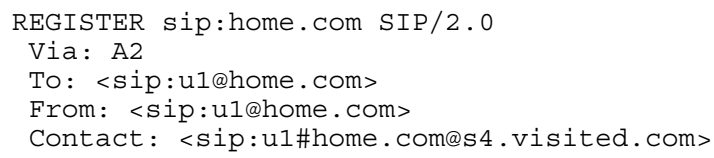

The local proxy, upon reception of the registration request from the mobile user, creates a new SIP REGISTER message and sends it to the local registrar (s4.visited.com), in order to execute the registration in the visited domain. Afterwards, it simply forwards the original REGISTER message to the home domain, without any modification.

The local registration is again a third-party procedure as the REGISTER message is generated by the outbound proxy, which has also to know the userís local IP address in order to build the right Contact header. 
The main fields of the local registration message are:

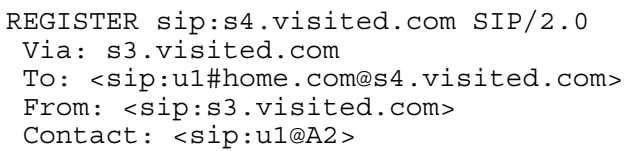

This approach has the advantage that it does not interfere with cryptographically signed registration requests, as the Contact field of the original REGISTER message is not modified by the local proxy. In this way, authentication between the mobile user and its home domain is not violated. However, modifications of the SIP User Agent and of the local DHCP server [3][10] are required to allow the mobile user to build ad-hoc REGISTER requests.

\section{c) Home Initiated}

This scenario (see Fig. 4) is another case of third-party local registration. This time, the home registrar, upon reception of the SIP REGISTER message of the mobile user, registers the user in the visited domain supplying its own credential. This approach has the fundament characteristic that the home registrar should act also as registering user agent, and thus both registered and registering parties belong to the same administrative domain. This scenario can be used in those cases where the home domain wants to have more control of its mobile users that are roaming.

The REGISTER message received by the home domain is in the form of:

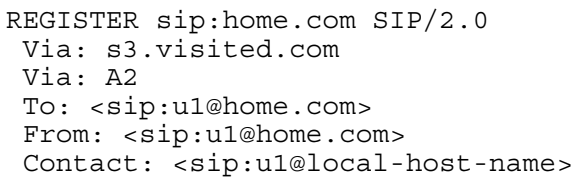

As usual, the Home Entry Proxy, once received the message, selects the registrar for the mobile user and forwards the following SIP message to it:

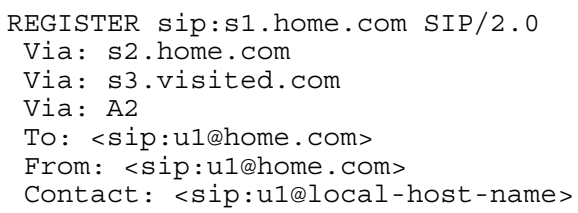

The Home Registrar uses the domain name supplied by the user in the Contact header in order to create the Register message for the registration in the visited domain. This can obviously work only if the mobile user supplies a domain name of the local access point rather than the relative IP address. The home registrar has to extrapolate the visited domain name from the ì local-host-nameî provided by the Contact header in the home registration message. Moreover, it has to create an identifier (u2) for the identification of the mobile user in the visited domain

The message for the registration in the visited domain is in the form of:

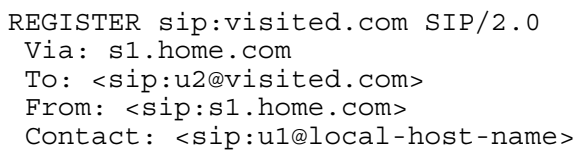


It is worth to notice that the home registrar can perform the Home Registration procedure only after the reception of the $200 \mathrm{OK}$ of the Local Registration, as the former depends on the successful of the latter.

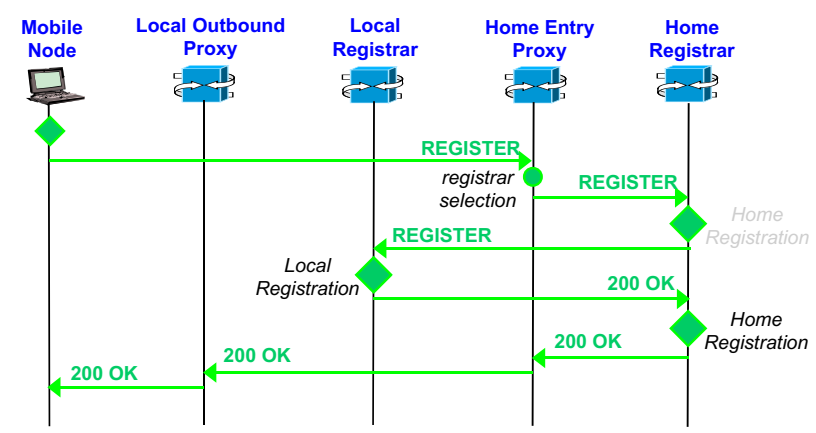

Fig. 4. Home-initiated Local Registration

\subsection{Dual Registration}

In this case (see Fig. 5), the mobile user sends two SIP REGISTER messages:

1) One to the local registrar, via multicast or the DHCP-configured outbound proxy, in order to execute the Local registration

2) The second one to the home domain in order to execute the Home registration The first message (local registration) has the following main fields:

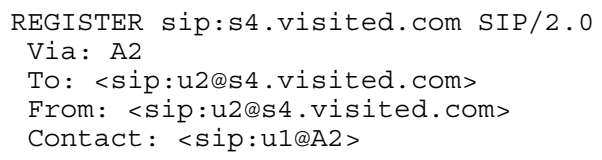

The local registration uses the canonical visitor name to avoid collisions (e.g. $\mathrm{u} 2=\mathrm{u} 1$ \#home.com).

The second message (home registration) has the following main fields:

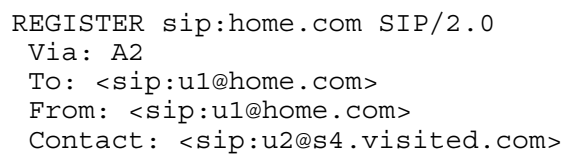

The home registration uses the local identifier and the name of the chosen local registrar.

This approach has the advantage that error handling is simplified, as both local and home registrations are fully separated. However the user should first execute the local registration and, only after the reception of the $200 \mathrm{OK}$, begin the home registration (Fig. 5). In order to reduce the time required for the registration, the REGISTER messages could be sent at the same time. The outbound proxy only forwards the outgoing messages, without modifying any fields. 


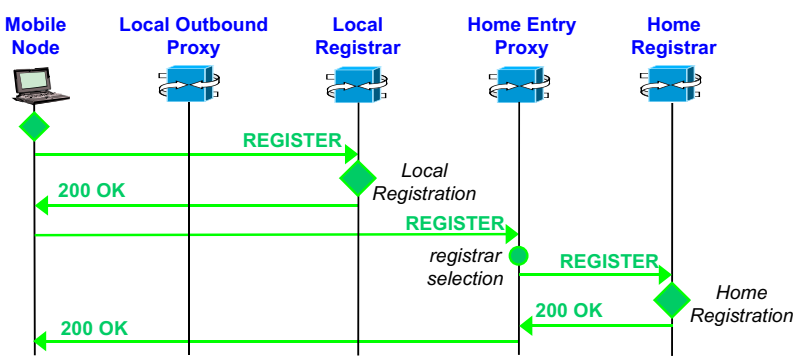

Fig. 5. Dual Registration

This approach requires two messages to be sent by the mobile user and this fact implies:

- inefficient use of the radio interface: the exchange of signalling messages between the user and the network is not minimized. This is strongly undesirable, particularly for bandwidth-constrained environments.

- changes in current SIP User Agent are required, as the mobile user must know the address of the local registrar and the new identifier (u2) that should be used in the Contact header of the message sent to the home registrar

Moreover, the mobile user must know all the information required to both registration procedures. Extensions $[3][10]$ are thus required at DHCP servers as well.

\section{Conclusions}

In this paper the problem of roaming within an all-IP wireless environment has been focused. The SIP architecture has been considered as control platform for the management of both user and terminal mobility. In particular we focused on the so-called SIP pre-call terminal mobility, that is the capacity for a user to be reachable when moving with its terminal amongst different administrative domains (or ISPs). Different SIP registration scenarios have been identified and described. For each scenario the message flow has been detailed, and the main advantages and drawbacks have been outlined. The proposed registration mechanisms can fit different call handling approaches and can be used to implement various service models. For example, different scenarios can refer to service models in which the service is controlled by the home domain, by the visited domain, or by both networks, according to user and/or service profile. We are currently working on the development of such roaming scenario in a real testbed. An interesting point for further works, is the performance evaluation of both signaling load and MS handover latency, by means of ad-hoc simulation studies or through a real implementation. 


\section{References}

1. J. Rosenberg, H. Schulzrinne et al. - ì SIP: Session Initiation Protocolî, IETF Request For Comments, RFC 3261, June 2002.

2. Charles E. Perkins ñ ì IP Mobility Supportî, IETF Request For Comments, RFC 2002, October 1996.

3. M. Cappiello, A. Floris, L. Veltri, ì IP Roaming Scenarios with AAA Supportî, IEEE International Communication Conference 2002 (ICC2002, New York, April/May 2002).

4. Elin Wedlund, Henning Schulzrinne - "Mobility Support Using SIP" ACM/IEEE, Internationl Conference on Wireless and Multimedia, WOWMOM, August 1999

5. H. Schulzrinne, E. Wedlund - ì Application-Layer Mobility Using SIPî, Mobile Computing and Communication Review, Volume 1, Number 2.

6. Dutta, F. Vakil, J.C Chen, Miriam Tauil, S. Baba, N. Nakajima, and Henning Schulzrinne "Application Layer Mobility Management Scheme for Wireless Internet " 3G Wireless 2001

7. Vakil, A. Dutta, J-C. Chen, M. Tauil, S. Baba, N. Nakajima, Y. Shobatake, H. Schulzrinne ì Supporting Mobility for Multimedia with SIPî, IETF Internet Draft, draft-itsumo-sipping-mobilitymultimedia-01.txt, July 2001 [expired].

8. 3GPP TS 23.228 - "IP Multimedia Subsystem (IMS); Stage 2 (Release 5)", 3rd Generation Partnership Project, Technical Specification Group Services and System Aspects (work in progress)

9. R. Droms - ì Dynamic Host Configuration Protocolî, IETF Request For Comments, RFC 2131, March 1997.

10. H. Schulzrinne - ì DHCPv4 Option for SIP Serversî, IETF Internet Draft, draft-ietf-sip-dhcp-06.txt, March 2002.

11. McAuley, S. Madhani, S. Baba, Y. Shobatake - ì Dynamic Registration and Configuration Protocol (DRCP)î, IETF Internet Draft, draft-itsumo-drcp-01.txt, July 2000 [expired].

12. H. Basilier, P. R. Calhoun, et al. ì AAA Requirements for IP Telephony/Multimediaî, IETF Internet Draft, draft-calhoun ñsip-aaa-reqs-04.txt, March 2002. 